\title{
An New Filtering Methods in the Wavelet Domain for Bowel Sounds
}

\author{
Zhang xizheng ${ }^{1}$, \\ ${ }^{1}$ School of Computer and Communication \\ Hunan Institute of Engineering \\ Xiangtan China
}

\author{
Rui Yuanqing ${ }^{2}$, Wang weixiong ${ }^{1}$ \\ ${ }^{2}$ School of Physics \\ South China University of Technology \\ Guangzhou ,China
}

\begin{abstract}
Bowel sounds signal (BS) is one of the important human physiological signals, analysis of the BS signal can then study gastrointestinal physiology and implement direct and effective diagnosis of gastrointestinal disorders. Use different threshold denoising methods denoising the original bowel sounds, simulated in the environment of MATLAB, then compared the denoising results and analyzed the advantages and disadvantages of those threshold denoising methods.
\end{abstract}

Keywords-bowel sounds; de-noising; wavelet transform; threshold

\section{INTRODUCTION}

Bowel sounds signal (BS) is the human digestive organs particularly the bowel movements and intestinal narrowing of energy in the intestinal wall pressure release form, which produces small vibration in the abdominal body surface. Through the sensing device of the abdominal skin vibration (ASV) system can capture these quivers. Analysis of the BS signal can then study gastrointestinal physiology and offer effective diagnosis for gastrointestinal disorders directly. However, there are many difficulties when extract BS signal from the data captured by ASV. As the body's own signal is weak, the signal is very susceptible to be contaminated by other signal source. In the case of sensor sensitivity and signal amplification and conditioning circuit performance is limited, the difficulties and key to extract the true BS signal is to reduce the extent of noise pollution or even eliminate the noise ${ }^{[1-5]}$.

To solve this difficult problem, the conventional method of signal filtering is often ineffective or powerless. Wavelet transform is a new signal processing tools which developed over a decade, because of its time and frequency domain also has good localization properties, multi-resolution analysis, "centralized" in nature and easy to combine with auditory and visual characteristics of human, therefore, wavelet is very suitable for non-stationary signals such as separation of signal and noise. The technology of BS signal filtering in wavelet domain is not mature, and left a lot of work and issues to be resolved. Specific to the country, it has not seen the relevant technical literature and reports appear. Therefore, from the theoretical and academic point of view, study the formation mechanism, signal characteristics and filtering techniques in wavelet domain of
BS this complex and highly unstable medical signal, can greatly enrich the theory of wavelet filtering itself, and is one of the most likely to occur theoretical breakthrough and technological innovation in the next several years ${ }^{[6-9]}$.

This paper using several methods to implement filtering for the collected BS signal in the wavelet domain, through qualitative and quantitative analysis, the optimal filtering method is proposed, with this kind of filtering method implement filtering can eliminate noise and retain the useful component effectively, and recovered the features of BS signal and can provide some help for the clinical diagnosis.

\section{BASIC THEORY OF WAVELET ANALYSIS}

\section{A. Continuous Wavelet Transform(CWT)}

$\forall f(t) \in L^{2}(R)$, the CWT of $f(t)$ is defined as ${ }^{[10,11]}$.

$$
W T_{f}(a, b)=|a|^{-1 / 2} \int_{-\infty}^{\infty} f(t) \overline{\psi\left(\frac{t-b}{a}\right)} d t, \quad a \neq 0
$$

Or use its inner product form:

$$
W T_{f}(a, b)=\left\langle f, \psi_{a, b}\right\rangle
$$

In the above formula $\psi_{a, b}(t)=|a|^{-1 / 2} \psi\left(\frac{t-b}{a}\right)$

In order to make inverse transform exist, $\psi(t)$ must meet the below requirement:

$$
C_{\psi}=\int_{-\infty}^{\infty} \frac{|\hat{\psi}(\omega)|^{2}}{|\omega|} d \omega<\infty
$$

In the above formula, $\hat{\psi}(\omega)$ is the FT of $\psi(t)$.

Then, the inverse transform is

$$
f(t)=C_{\psi}^{-1} \int_{-\infty}^{\infty} \int_{-\infty}^{\infty} \psi_{a, b}(t) W T_{f}(a, b) d b \frac{d a}{|a|^{2}}
$$

In MATLAB, we can use the function of $c w t$ to implement CWT for continuous signal ${ }^{[2]}$. 


\section{B. Discrete Wavelet Transform(DWT)}

In practice, especially when implemented on a computer, continuous wavelet transform must be discrete. So, it is necessary to discuss the discretization of continuous wavelet $\boldsymbol{\Psi}_{a, b}(t)$ and continuous wavelet transform $W_{\mathrm{f}}(\mathrm{a}, \mathrm{b})$. Need to stress that the above-mentioned discretization is towards continuous scale parameter $a$ and continuous translation parameter $b$, but not time parameter $t$.

In the continuous wavelet, consider the function:

$$
\boldsymbol{\Psi}_{a, b}(t)=|a|^{-1 / 2} \boldsymbol{\Psi}\left(\frac{t-b}{a}\right)
$$

And $b \in R, a \in R^{+}, a \neq 0, \boldsymbol{\Psi}$ is allowed, for convenience, $a$ must be limited to positive in discretization, and now the compatibility requirement can be represented as

$$
C_{\Psi}=\int_{0}^{\infty} \frac{|\hat{\boldsymbol{\Psi}}(\bar{\omega})|}{|\bar{\omega}|} \mathrm{d} \bar{\omega}<\infty
$$

Generally, the discretization formula of continuous wavelet transform scale parameter $a$ and translation parameter $b$ can be taken as $a=a_{0}^{j}$ and $b=k a_{0}^{j} b_{0}$, and $j \in Z$,expansion step $a_{0} \neq 1$ is a fixed value, for convenience, always assume that $a_{0}>1$.So the corresponding discrete wavelet function $\boldsymbol{\Psi}_{j, k}$ can be writed as

$$
\boldsymbol{\Psi}_{j, k}(t)=a_{0}^{-j / 2} \boldsymbol{\Psi}\left(\frac{t-k a_{0}^{j} b_{0}}{a_{0}^{j}}\right)=a_{0}^{-j / 2} \boldsymbol{\Psi}\left(a_{0}^{-j / 2} t-k b_{0}\right)
$$

And discretization wavelet transform coefficient can be expressed as

$$
C_{j, k}=\int_{-\infty}^{\infty} f(t) \boldsymbol{\Psi}_{j, k}^{*}(t) \mathrm{d} t=\left\langle f, \boldsymbol{\Psi}_{j, k}\right\rangle
$$

The corresponding reconstruction formula is

$$
f(t)=C \sum_{-\infty}^{\infty} \sum_{-\infty}^{\infty} C_{j, k} \boldsymbol{\Psi}_{j, k}(t)
$$

$C$ is constant independent of $f(t)$.

In MATLAB, the function of $d w t$ and $d w t 2$ can use for DWT.

\section{Multi-resolution Analysis}

In order to understand multi-resolution, we will choose a decomposition of three to clarify it; the wavelet decomposition tree is shown as Figure 1.

It is clear from Figure 1 that the further decomposition of multi-resolution analysis is towards low frequency, while not consider the part of high frequency. The relation of decomposition is $\mathrm{S}=\mathrm{A} 3+\mathrm{D} 3+\mathrm{D} 2+\mathrm{D} 1$. In addition to stress that here is one level decomposition, if it need further decomposition, the part of low frequency A3 can bread down into part of low frequency A4 and part of high frequency D4, and so on.

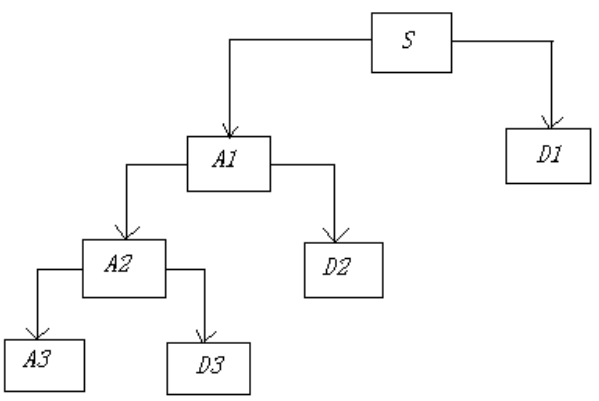

Figure 1 three multi-resolution analysis tree

In order to understand multi-resolution analysis, we must firmly grasp the point that the ultimate purpose of decomposition is seek to construct a orthogonal wavelet base which highly approach the space of $\mathrm{L}^{2}(\mathrm{R})$ in frequency. These orthogonal wavelet bases of different frequency resolution are equal to band pass filter of different bandwidth. As can be seen from Figure 1, multi-resolution analysis on further decomposition of low frequency space, which made the frequency resolution, became more and more high.

\section{PRINCIPLE OF WAVELET THRESHOLD DENOISING}

\section{A. The Process of One-dimensional Signal Denoising}

(1)Wavelet decomposition of one-dimensional signal. Choose a wavelet and determine the level of a wavelet transformn $N$, then implement $N$ layers wavelet decomposition of signal $S$.

(2)Threshold quantization of high-frequency coefficient of wavelet transform. Selecting a threshold implement soft threshold quantization for high-frequency coefficient of 1 to $\mathrm{N}$ layer.

(3)Reconstruction of one-dimensional wavelet. Implement wavelet reconstruction for low-frequency of NO.N and high-frequency of 1 to $\mathrm{N}$ layer which experienced the process of quantization.

In these three steps, the most critical is how to select the threshold and how to quantity the threshold. To some extent, it is directly related to the quality of the signal denoising ${ }^{[10-12]}$.

\section{B. Principle of Threshold Selection in Wavelet Denoising} The following threshold can be chosen: 
tptr=' rigrsure' is an adaptive threshold selection based Stein's unbiased likelihood estimation principle. For a given threshold $t$, getting its likelihood estimation first, then minimize the nonlikelihood $t$, so the threshold has been obtained. It is a software threshold estimator.

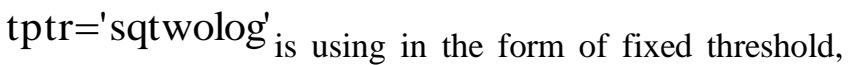
the threshold generated is " $\operatorname{sqrt}(2 * \log (\operatorname{length}((\mathrm{X})))$ "

$\operatorname{tptr}=$ 'heursure' is integration of the first two threshold, and also the best predict variable threshold selection. If the signal to noise ratio is small, SURE estimation will have lots of noise. If this occurs, we can use this fixed threshold.

tptr='minimaxi' is also using a fixed threshold, it produces an extremum with minimum mean square error, but not without error. Statistically, this maximum principle is used for designing estimator. Because the signal needed de-noising can be seen similar to the estimation of unknown regression function, this extreme value estimator can realize minimize of maximum mean square error for a given function ${ }^{[12]}$.

\section{Simulation ANALYSIS}

In the MATLAB environment, first of all, implement low-bass filtering for original collection BS signal. As far as the results of foreign literature, BS spectrum is distributed in $50 \sim 1500 \mathrm{~Hz}$ frequency range. As the acquisition process of original signal can not prevent some other band noise interference, it is necessary to implement low-pass prefiltering for the original signal; this can greatly enhance the effect of noise elimination, and at the same time reduce the burden on the wavelet filter. Then, use four threshold methods of the function wden and the default threshold denoising of the function wdencmp. Select the waveletof $d b 4$ and the decomposition level of 5 .

Denoising command under MATLAB is:

Four threshold denoising code:

xd1=wden (y,'rigrsure','s','one',5,'db4');

xd2=wden(y,'heursure','s','one',5,'db4');

xd3=wden (y,'sqtwolog','s','one',5,'db4');

xd4=wden(y,'minimaxi','s','one',5,'db4');

The default threshold denoising method code:

[thr,sorh,keepapp]=ddencmp('den','wv',y);

xd5=wdencmp('gbl',y,'db4',5,thr,sorh,keepapp); $\mathrm{xd} 1 \sim \mathrm{xd} 5$ are denoising results, the results are shown as Figure (d) Figure (h). And Figure (a) is the original signal, Figure (b) is its spectrum and Figure (c) is the low-pass filtering result.

From the above graph you can see, the spectrum of original signal distributed in $0 \sim 4000 \mathrm{~Hz}$, the main component distributed in $0 \sim 2000 \mathrm{~Hz}$, and there is a maximum at $228 \mathrm{~Hz}$, that verify the normal BS signal have a maximum peak between $160 \mathrm{~Hz}$ and $260 \mathrm{~Hz}^{[3]}$.

Compare the above chart with Figure (a), this signal after low-pass filtered slightly different than the original signal, because the main spectrum of original signal distributed in $0 \sim 2000 \mathrm{~Hz}$, while the low-pass filter retain the signal spectrum range of $0 \sim 1500 \mathrm{~Hz}$, so get this result.

Compare Figure (d) Figure (g) with Figure (c), and Figure (d) $\sim$ Figure $(g)$ are the results of the four threshold denoising. Compared to the signal through low-pass prefiltered, the four kinds of threshold methods have removed some noise. Compare the Figure (d) and Figure (g) with Figure (e) and Figure (f), the naked eye can see that the first two denoising are better than the latter two, that's to say that the rigrsure and minimaxi threshold method can effectively remove noise while retain the characteristics of $\mathrm{BS}$. The denoising results are very similar with the results of previous studies. While the heursure and sqtwolog threshold denoising method at the same time may remove some of the useful component.

Compare the above chart with Figure (c), we can see that the default threshold denoising method have removed some noise, but the denoising effect is not significant and can not recover bowel sounds better.

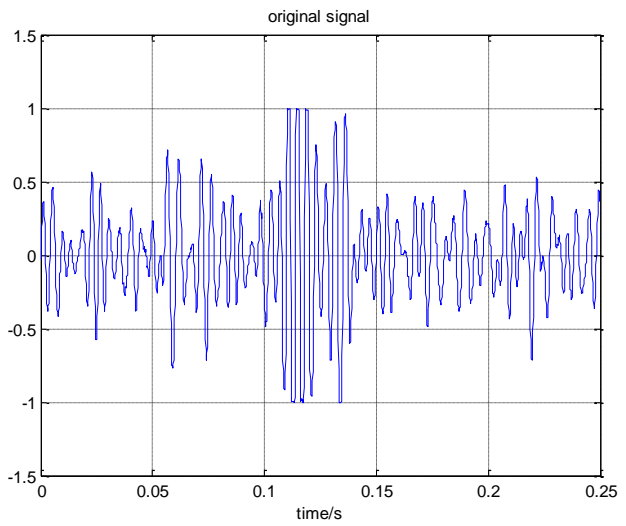

Figure (a)

Where $y$ is a signal through low-pass filter. 


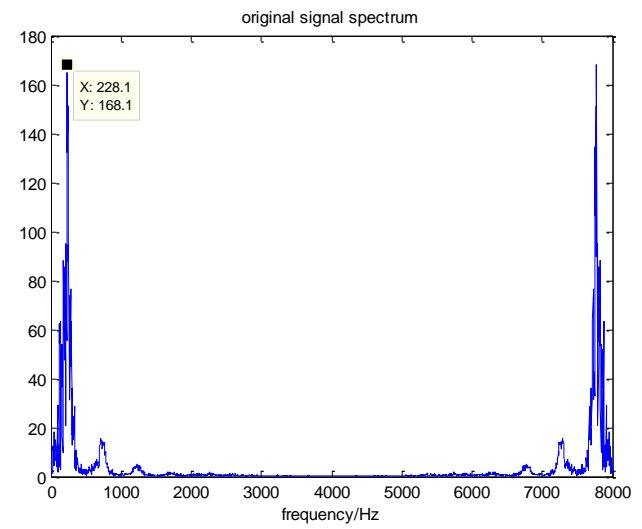

Figure (b)

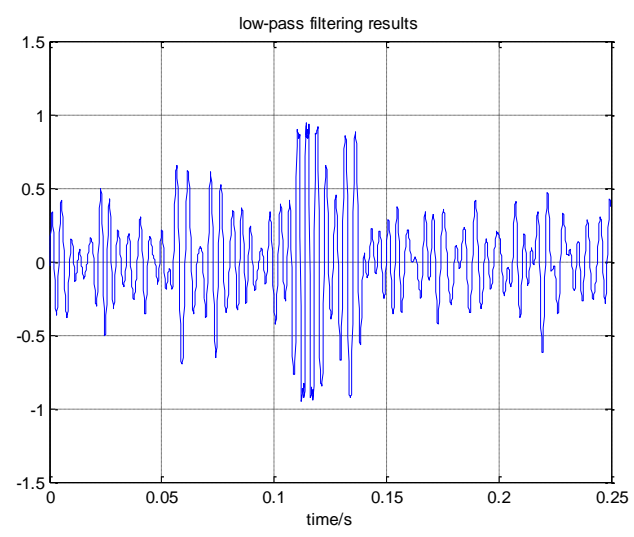

Figure (c)

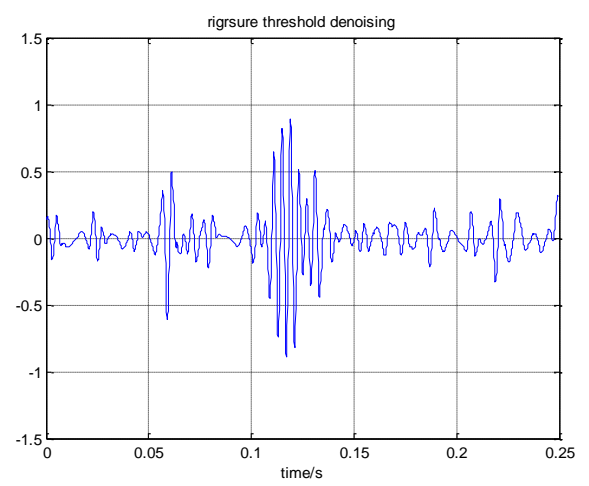

Figure (d)

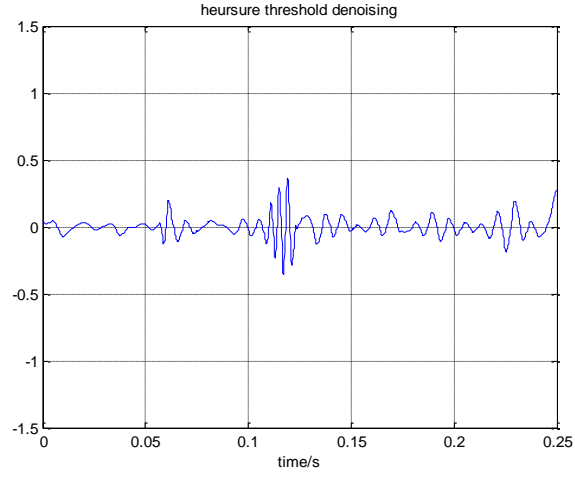

Figure (e)

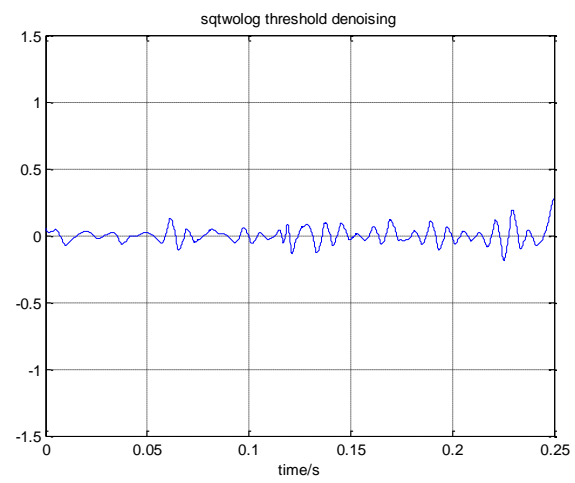

Figure (f)

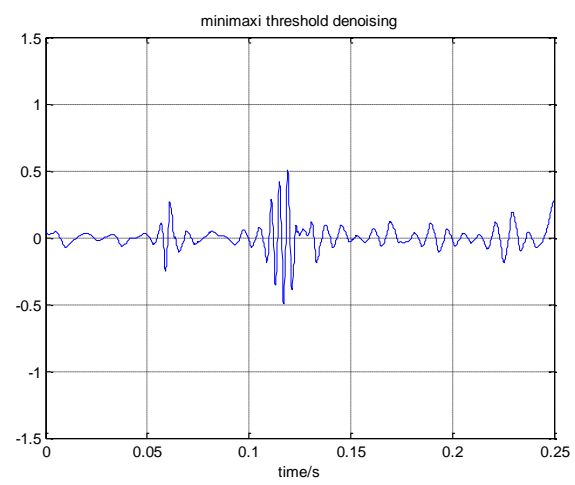

Figure (g)

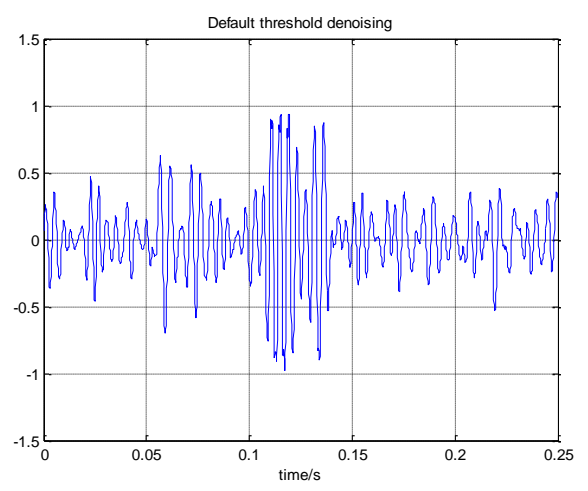

Figure (h) 
In order to represent the denoising effect more accurately, we can calculate the signal to noise ratio and the root mean square error for the four types of threshold denoising and the default threshold denoising ${ }^{[4]}$. Use the original signal $x(n)$ and the filtered signal $x(n)^{\prime}$, we can define the signal to noise ratio as ${ }^{[15]}$ :

$$
\mathrm{SNR}=10 \log \left[\frac{\sum_{n} x(n)^{2}}{\sum_{n}\left[x(n)-x(n)^{\prime}\right]^{2}}\right]
$$

The root mean square error of original and denoised signal is defined as:

$$
\operatorname{RMSE}=\sqrt{\frac{1}{n} \sum_{n}\left[x(n)-x(n)^{\prime}\right]^{2}}
$$

The higher signal to noise ratio, the smaller the root mean square error, the closer the denoising signal with the original signal, the better the denoising effect ${ }^{[13-5]}$.

Shown in Table 1, we can see SNR and RMSE which used $d b 4$ wavelet.

\begin{tabular}{|c|c|c|c|c|c|}
\hline & $\begin{array}{l}\text { rsure }^{\text {rig }} \\
\text { thr } \\
\text { eshold }\end{array}$ & \begin{tabular}{l}
\multicolumn{1}{c}{ heu } \\
rsure \\
threshold
\end{tabular} & $\begin{array}{l}\begin{array}{c}\text { sqt } \\
\text { wolog }\end{array} \\
\text { thre } \\
\text { shold }\end{array}$ & $\begin{array}{l}\operatorname{mini} \\
\text { maxi } \\
\text { threshold }\end{array}$ & $\begin{array}{l}\text { ault }{ }^{\text {def }} \\
\text { thr } \\
\text { eshold }\end{array}$ \\
\hline $\begin{array}{l}{ }_{\mathrm{R}(\mathrm{db})}^{\mathrm{SN}} \\
\end{array}$ & $471^{3.5}$ & $926^{0.7}$ & $\begin{array}{ll} & 0.22 \\
83 & \end{array}$ & $\begin{array}{ll} & 1.17 \\
93 & \end{array}$ & $3385^{16 .}$ \\
\hline 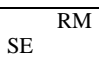 & $093^{0.2}$ & $875^{0.2}$ & $\begin{array}{ll}68 & 0.30\end{array}$ & $\begin{array}{ll}49 & 0.27 \\
49 & \end{array}$ & $480^{0.0}$ \\
\hline
\end{tabular}

Table 1 the SNR and RMSE after denoising $(d b 4)$

If use the symlet 4 wavelet, the SNR and RMSE after denoising are shown in Table 2. But the denoising results are omitted.

\begin{tabular}{|c|c|c|c|c|c|}
\hline & \begin{tabular}{l}
\multicolumn{1}{c}{$r i g$} \\
rsure \\
threshold
\end{tabular} & \begin{tabular}{l}
\multicolumn{1}{c}{ heu } \\
rsure \\
threshold
\end{tabular} & $\begin{array}{l}\begin{array}{c}\text { sqt } \\
\text { wolog }\end{array} \\
\text { thre } \\
\text { shold }\end{array}$ & $\begin{array}{l}\operatorname{mini} \\
\text { maxi } \\
\text { threshold }\end{array}$ & $\begin{array}{l}\text { ault def } \\
\text { thr } \\
\text { eshold }\end{array}$ \\
\hline $\begin{array}{l}{ }_{\mathrm{R}(\mathrm{db})}^{\mathrm{SN}} \\
\end{array}$ & $583^{1.9}$ & $806^{0.6}$ & $\begin{array}{ll} & 0.19 \\
85 & \end{array}$ & $\begin{array}{ll} & 1.07 \\
26 & \end{array}$ & 490716. \\
\hline $\begin{array}{ll} & \text { RM } \\
\text { SE } & \end{array}$ & $514^{0.2}$ & $912^{0.2}$ & $\begin{array}{ll}78 & 0.30 \\
78\end{array}$ & $\begin{array}{ll} & 0.27 \\
83 & \end{array}$ & $472^{0.0}$ \\
\hline
\end{tabular}

Table 2 the SNR and RMSE after denoising (sym4)

SNR and RMSE calculated above is to test the effect of wavelet filtering, so the original signal $x(n)$ is equal to signal $y$ after low-pass filtered, while $x(n)^{\prime}$ ' is equal to wavelet denoising signal $x d$.

From Table 1 and Table 2 shows, four threshold denoising and default threshold denoising methods are improved SNR, and have removed some noise. From the denoising effect, the principle of rigrsure threshold and minimaxi threshold denoising are better than the other three methods, because the two denoised SNR higher than heursure and sqtwolog denoising methods and its RMSE are smaller. While the default threshold method have the highest
SNR and smallest RMSE, however, we can see from the waveform after denoising that the denoising effect is not obvious, there is no effective removal of noise. While the heursure threshold and sqtwolog threshold principle will remove useful signal as noise. If there is high frequency information signal, the principle of the two threshold denoising results will not be satisfactory. Compare Table 1 with Table 2, we can be seen that using the db wavelet and the sym wavelet, the denoising effect of the former is better than the latter.

\section{CONCLUSION}

From the simulation analysis, the wavelet transform in signal denoising in particular nonstationary signals, can effectively remove noise and improve SNR. With regard to the bowel sounds which complex and highly nonstationary, wavelet transform denoising can play the advantages compared to traditional denoising, wavelet can better demonstrate its advantages. From the simulation results, we can obtain that use the principle of rigrsure threshold and minimaxi threshold can effectively reduce noise, and can retain a useful component of bowel sounds. Use heursure and sqtwolog threshold to denoising, it will remove a useful component of bowel sounds at the same time, this method more harm than good. Use the default threshold method to denoising can not effectively remove the noise, unable to obtain the signal characteristics of bowel sounds.

\section{ACKNOWLEDGEMENTS}

The authors are grateful to the anonymous reviewers and to the Natural Science Foundation of Hunan Province for supporting this work through research grant JJ076111, and the Student Innovation Programme of Hunan Province through research grant 513 .

\section{REFERENCES}

[1] G. Jianbo, H. Sultan, H. Jing, T. Wenwen, "Denoising Nonlinear Time Series by Adaptive Filtering and Wavelet Shrinkage: A Comparison", IEEE Signal Processing Letters, 2010, vol.17, no.3, pp.237-240.

[2] G. Zhexue, C.Zhongsheng, "Matlab Time-frequency Analysis and Its Application (Second Edition)," Beijing: Posts \& Telecom Press. 2009.

[3] M.Kania, M.Fereniec, and R. Maniewski, "Wavelet denoising for multi-lead high resolution ECG signals", Measurement Science review, 2007, vol.7., no.1, pp. 30-33.

[4] Zhang Hehua. Bowel sounds acquisition system design and the signal analysis methods research. Master's degree thesis of Third Military Medical University. 2009.5.1.

[5] Wu Wei,Cai Peisheng,"Wavelet denoising simulation based on MATLAB. Signal and Electronic Engineering,2008,vol. 6, no.3, pp.220-222.

[6] Qin Yahui,Feng Jinghui,Chen Liding, "Study of signal denoising methods based on wavelet transform. Information Technology, 2010, vol.5, no.2, pp.53-55.

[7] C.Dimoulas ,G.Kalliris ,G.Papanikolaou ,V.Petridis ,A.Kalampakas,"Bowel-sound pattern analysis using wavelets and neural networks with application to long-term, unsupervised, gastrointestinal motility monitoring", Expert Systems with Applications, 2008, vol.38, no.1, pp.26-41. 
[8] A. Borsdorf, R. Raupach, T. Flohr, and J. Hornegger, "Wavelet Based Noise Reduction in CT-Images Using Correlation Analysis," IEEE Transactions on Medical Imaging, 2008, vol.27, no.12, pp.16851703.

[9] R. Ranta, V. Louis-Dorr, Ch. Heinrich, D. Wolf, "Guillemin Digestive activity evaluation by multi-channel abdominal sounds analysis", IEEE Transactions on Biomedical Engineering, in press.

[10] Z. Hehua, W. Baoming, Z. Lianyang, et.al. "Study on Adaptive Filter and Methods of Bowel Sounds Features Extraction", Journlal of Medical Equipments, 2009, vol.26, no.3, pp.58-62.

[11] C.Fu, C, Lifei, "Data mining to improve personnel selection and enhance human capital: A case study in high-technology industry", Expert Systems with Applications, 2008, vol.34, no.1, pp. 280-290.

[12] R. Ranta, R. Louis-Dorr, V. Heinrich, et.al., "Digestive Activity Evaluation by Multichannel Abdominal Sounds Analysis", IEEE Transactions on medical Engineering, 2010, vol.57, no.6, pp.15071519.

[13] D.Matsiki, "Wavelet-based analysis of nocturnal snoring in apneic patients undergoing polysomnography", Conf Proc IEEE Eng Med Biol Soc, Thessaloniki, Greece, 2007, pp.1912-1915.
[14] I. K. Kitsas, "Classification of transmembrane segments in human proteins using wavelet-based energy", Proc IEEE Eng Med Biol Soc Conf, hessaloniki, Greece, 2007, pp.1225-1228.

[15] W. Wei, Z.Z. Ge, H. Lu, et al. , "Purgative bowel cleansing combined with simethecone improves capsule endoscopy imaging", American Journal of Gastroenterol, 2008, vol.103, no.1, pp.77-82.

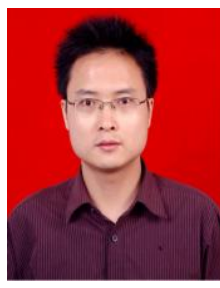

Xizheng Zhang received the B.S. degree in control engineering and the M.S. degree in circuits and systems in 2000 and 2003, respectively, from Hunan University, Changsha, China, where he is currently working toward the $\mathrm{Ph} . \mathrm{D}$. degree with the College of Electrical and Information Engineering.

$\mathrm{He}$ is also with the School of Computer and Communication, Hunan Institute of Engineering, Xiangtan, China. His research interests include industrial process control and artificial neural networks. 